
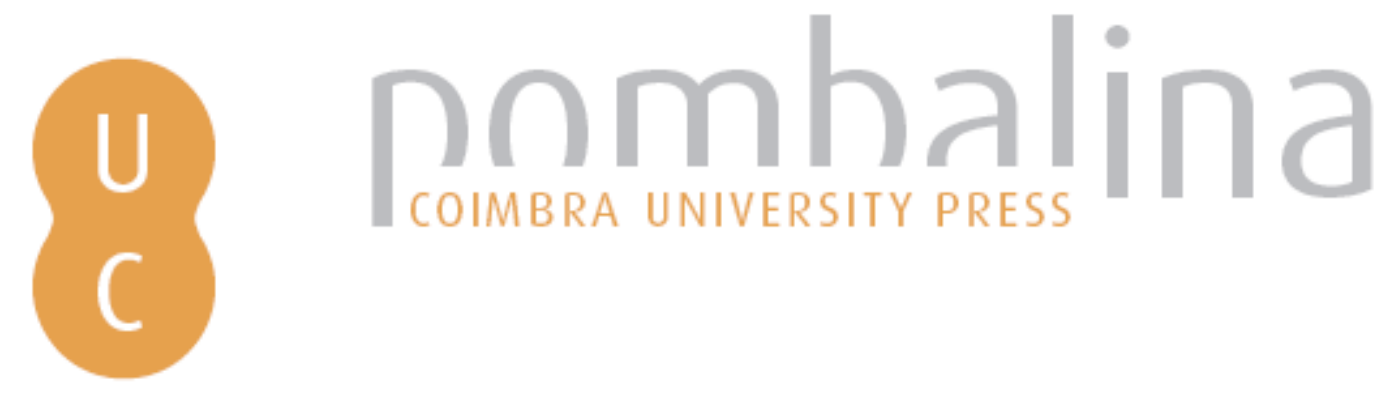

\title{
La investigación en el resumen documental: dimensiones, enfoques y técnicas
}

\author{
Autor(es): $\quad$ Alonso, Mónica Izquierdo
}

Publicado por: Imprensa da Universidade de Coimbra

URL

persistente: URI:http://hdl.handle.net/10316.2/31884

DOI: $\quad$ DOI:http://dx.doi.org/10.14195/978-989-26-0869-3_5

Accessed : $\quad$ 26-Apr-2023 12:12:34

A navegação consulta e descarregamento dos títulos inseridos nas Bibliotecas Digitais UC Digitalis, UC Pombalina e UC Impactum, pressupõem a aceitação plena e sem reservas dos Termos e Condições de Uso destas Bibliotecas Digitais, disponíveis em https://digitalis.uc.pt/pt-pt/termos.

Conforme exposto nos referidos Termos e Condições de Uso, o descarregamento de títulos de acesso restrito requer uma licença válida de autorização devendo o utilizador aceder ao(s) documento(s) a partir de um endereço de IP da instituição detentora da supramencionada licença.

Ao utilizador é apenas permitido o descarregamento para uso pessoal, pelo que o emprego do(s) título(s) descarregado(s) para outro fim, designadamente comercial, carece de autorização do respetivo autor ou editor da obra.

Na medida em que todas as obras da UC Digitalis se encontram protegidas pelo Código do Direito de Autor e Direitos Conexos e demais legislação aplicável, toda a cópia, parcial ou total, deste documento, nos casos em que é legalmente admitida, deverá conter ou fazer-se acompanhar por este aviso. 
Maria Manuel Borges

Elias Sanz Casado

Coordenação

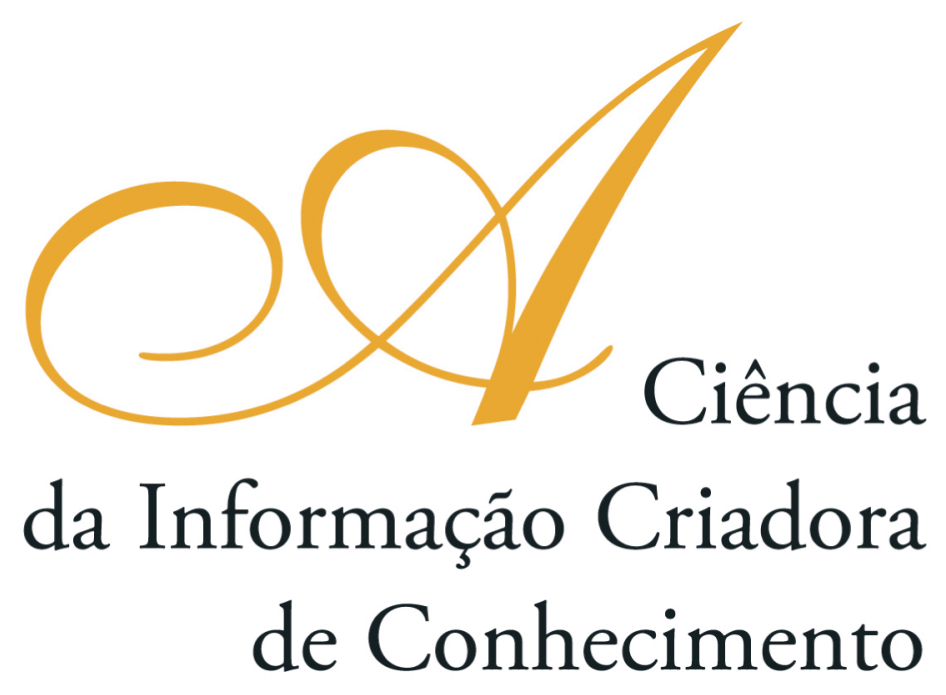

Vol. I I

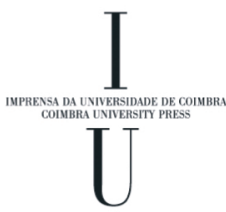

- COImbra 2009 


\title{
La investigación en el Resumen Documental: \\ DIMENSIONES, ENFOQUES Y TÉCNICAS
}

\author{
Mónica Izquierdo Alonso \\ Universidad de Alcalá (Espanha)
}

\section{Resumen}

Se presenta un estudio diacrónico del fenómeno del resumen documental y de la teoría general resumidora con el objetivo de analizar las diferentes propuestas teórico- metodológicas del hecho resumidor, desde una perspectiva sistémica y comparada tanto de los objetos de estudio como de las variables de análisis implicadas en esta realidad documental. Para ello, se realiza una revisión de las perspectivas y propuestas de análisis, que interesan a los distintos teóricos del resumen, bajo una pluralidad de técnicas y enfoques (cognitivos, lingüísticos, comunicativo-sociales, didácticos, etc.), cada uno con distintos niveles de abstracción teórico-metodológica y grado de aplicación. El fin último será trazar un pequeńo mapa de los estudios del resumen documental: a) que nos señale las rutas investigadoras con sus incidencias, convergencias y divergencias; b) que nos indique cómo se va ampliando el marco conceptual y metodológico de aquel, paralelamente a la evolución de la propia Ciencia de la Documentación, e incluyendo nuevas variables de análisis que enriquecen y abren nuevas posibilidades en el área de la representación documental; y c) que nos permita identificar las áreas de interconexión así como las debilidades y fortalezas de los diversos enfoques y técnicas resumidoras, con el objetivo de mejorar el estatus científico del resumen documental y ampliar su realidad investigadora a nuevas dimensiones y retos.

\section{Introducción}

Los estudios de resumen documental han evolucionado de un modo extraordinario en las dos últimas décadas con el objetivo de configurar un espacio de conocimiento propio en las Ciencias Documentales: intentando delimitar espacios conceptuales, definir marcos y modelos operativos, diseñar estrategias eficaces de representación documental y resolución de problemas para mejorar la práctica resumidora, etc. En este sentido, se han desarrollado diferentes enfoques de análisis para esta operación de tratamiento documental de contenido desde una doble dimensión: como producto textual, considerando sus diversas perspectivas, y desde su faceta procesual, aislando los factores determinantes de la actividad resumidora bajo marcos teóricos y operativos cada vez más amplios y dinámicos. Las diversas aproximaciones han tendido a priorizar determinados elementos: el texto, la figura del resumidor y los procesos cognitivos, el fenómeno de la recepción del usuario desde un contexto social, la figura del resumidor y la adquisición de competencias resumidoras, etc. Todas estas propuestas están aún en proceso de consolidación debido quizás a la propia juventud de la Documentación como disciplina y al carácter multidimensional del objeto de estudio del propio resumen documental, en el que confluyen las ciencias del lenguaje, las ciencias sociocognitivas, las ciencias de la comunicación y las aplicaciones tecnológicas, entre otras. Sin 
embargo, los estudios sobre resumen documental van consolidando sus espacios de reflexión y praxis, demarcando poco a poco su propio núcleo epistemológico dentro del tratamiento documental de contenido, nutriéndose con un importante corpus de trabajos teóricos y aplicados. El problema radica en que los distintos modelos han delimitado su objeto de estudio desde parámetros y técnicas diversas, sobre todo en los modelos lingüísticos, ofreciendo visiones parciales de un campo de estudio complejo por su interdisciplinariedad. El objetivo final sería encontrar puntos de encuentro entre los diversos enfoques en aras a lograr una sistematización global: una teoría general del resumen y la actividad resumidora documental. En el plano conceptual y epistemológico sigue habiendo muchos caminos por explorar. Necesitamos dotarnos de un metalenguaje propio que refiera al hecho resumidor de forma coherente, sobre todo si tenemos en cuenta las inferencias de otras disciplinas que se ocupan del resumen en su dimensión de género textual diferenciado: como las ciencias cognitivas, desde la comprensión lectora; la lingüística aplicada, desde el análisis del discurso o la ingeniería lingüística y el procesamiento automático de textos. Del mismo modo, en la práctica profesional urge generar modelos resumidores eficaces, adaptados a los diversos ámbitos de especialidad y a los contextos de las distintas plataformas documentales y servicios de resúmenes.

Haremos un pequeño recorrido por cada una de los enfoques del resumen documental con el fin de mostrar cómo han ido surgiendo los diversos planteamientos en torno a una actividad eminentemente práctica que en un principio estaba lejos de ser algo teorizable.

\section{Objetivos}

El resumen documental es un acto social de comunicación con una función meta-representadora. Destacamos su dimensión compleja y poliédrica tanto en la naturaleza de su objeto, el texto desde su caracterización semiótica, como desde su actividad resumidora en la que inciden múltiples factores. Así pues en este estudio pretendemos:

- Acercarnos al fenómeno multidisciplinar de resumen documental desde diferentes perspectivas y modelos (textual, psicolingüístico, social y comunicativo), con el fin de sistematizar y mejorar el estatus científico de los estudios del resumen documental.

- Revisar las diferentes propuestas de análisis de los teóricos del resumen valorando las aportaciones más significativas así como sus limitaciones.

- Dibujar un pequeño mapa de los estudios del resumen documental que nos indique cómo se va ampliando el marco conceptual y metodológico de aquel, paralelamente a la evolución de la propia Ciencia de la Documentación.

- Reflexionar sobre la necesidad y pertinencia de investigar de un modo sistemático en resumen documental para fomentar nuevas hipótesis e iniciativas que den respuesta válida a los problemas planteados por la representación documental y la recuperación de información. 


\section{Tendencias en los estudios del resumen documental: enfoques metodológicos y perspectivas}

Las teorías sobre el resumen documental son bastante recientes. Es a partir del último tercio del siglo pasado cuando surge un interés por analizar de un modo sistemático las diferentes vertientes del resumen documental, reafirmándose este tipo de estudios, con carácter propio, dentro del tratamiento documental de contenido. No obstante, la operación de resumir es tan antigua como la necesidad humana de esquematizar y sintetizar el pensamiento con el fin de asimilarlo, recordarlo o almacenarlo. Tenemos muestras de resúmenes ya en la antigua Mesopotamia, Grecia, Roma y en la Edad Media. Sin embargo, en nuestra cultura occidental, su implantación social no llegará hasta el siglo XVII, paralela al desarrollo de las revistas científicas, y ligada a la primera revista de resúmenes: el Journal des $S_{\text {çavants }}{ }^{1}(1665)$. Posteriormente, la actividad del resumen científico se irá reforzando con el desarrollo de la Documentación como disciplina. Actualmente, los estudios de resumen se van consolidando desde la integración de teorías de disciplinas anexas y la apertura a nuevos modelos y técnicas de análisis.

Agruparemos los estudios teóricos del resumen documental en cuatro enfoques que hemos de considerar como perspectivas alternativas y, en muchos casos, complementarias:

- Enfoque textual: centrado en el texto

- Enfoque psicolingüístico: centrado en el proceso

- Enfoque sociocultural: centrado en el usuario y la comunidad discursiva

- Enfoque comunicativo-interaccional

\subsection{Enfoque del resumen centrado en el producto/texto}

El enfoque textual se fundamenta en la consideración del resumen documental como una operación intertextual. Esta orientación defiende que es necesario comprender la naturaleza del propio concepto del texto y para ello se apoyará en distintos modelos lingüísticos: desde los más puramente semánticos, hacia modelos tomados de la lingüística textual, avanzando hacia perspectivas semióticas y a consideraciones pragmático-comunicativas más dinámicas. Sin embargo esta perspectiva, que toma como referente al texto objeto y texto/resumen, resulta insuficiente si no se completa con una reflexión orientada al proceso resumidor que tome en cuenta las distintas estrategias y meta-estrategias que confluyen en las fases de reconocimiento, representación documental y la de revisión del propio resumen, así como el contexto comunicativo

${ }^{1}$ Tal y como señala el profesor López Yepes (1981:128), la técnica del resumen nace con el nuevo concepto de ciencia moderna y de la comunidad científica y se erige en el vehículo más idóneo de la actividad científico-informativa; y ello sobre un soporte determinado nacido al calor de los mismos principios inspiradores de la ciencia moderna: la publicación periódica de carácter científico. En el siglo XIX, y cuando ya se empezaba a tomar conciencia de un nuevo fenómeno relacionado con las publicaciones primarias —la explosión documental—, continúa la proliferación de revistas de resúmenes, iniciándose la tendencia hacia el desarrollo de publicaciones especializadas. 
en el que se crea el texto objeto de análisis y la situación comunicativa para la que se diseña y evalúa el resumen documental.

Dentro de este enfoque podemos advertir varias aproximaciones:

a) Las aproximaciones semánticas, como su propio nombre indica, destacan la importancia del significado en el resumen, de su acceso en las tareas de comprensión lectora y en su interpretación posterior tras la fase de redacción del resumen. Para los autores que se inscriben en esta perspectiva el resumen consiste en una representación exacta del contenido semántico del documento original, sin distorsiones de significado (Borko\&Bernier, 1975; Cremmins 1985, Rowley 1988, Lancaster 1996). Estos enfoques enlazarán, a partir de los ańos 90, con las teorías del resumen centradas en la macroestructura semántica desde las bases de la lingüística textual.

b) El enfoque semiótico considera el proceso de resumen, al igual que todo el tratamiento documental de contenido, como representación entre signos (el objeto que se resume y el resumido o resumen). Izquierdo Arroyo (1991, 1993) es uno de los máximos exponentes de esta línea y asume el resumen, como proceso interactivo de comunicación contextualizada y transducción/transcodificación semiótica. El autor reivindica la existencia de una rama general, la semiótica documental, como espacio integrador y vertebrador de los procesos de meta-representación. Desde una posición más avanzada, y a partir de posicionamientos pragmáticos, toma como punto de referencia del tratamiento documental de contenido la función del objeto semiótico [objeto en situación], determinada tanto por los elementos formales como de contenido del propio objeto, y adaptada a los objetivos de la situación comunicativo-documental. Desde estos valores semióticos conecta con la noción de intertextualidad como espacio de representación documental en el que los objetos semióticos son considerados como productos que forman parte del sistema de una comunidad discursiva con sus prácticas sociales (incluye aquí los esquemas epistemológicos o de dominio). Por tanto, la consideración de los determinantes contextuales es fundamental para entender la intertextualidad como mecanismo facilitador de las relaciones entre textos-símbolos en contextos interactivos de comunicación o procesos de semiosis social.

\section{c) Generativismo estructural: macroestructuras y resumen}

Estos autores trasladan la distinción de la gramática generativa entre estructura profunda y superficial al ámbito del texto y por extensión al del resumen. Partiendo de los postulados de Van Dijk, (1978, 1980, 1983) plantean la unidad del texto como una jerarquización a distintos niveles, locales y globales, desde la consideración de tres planos estructurales: superestructuras, macroestructuras y superestructuras. Asumen la macroestructura como una estructura semántica de síntesis y los resúmenes como reproducción o expresión de la macroestructura global de un texto (Pinto Molina, 1992, 1996; Moreiro 1993). Así, en sus metodologías resumidoras los autores destacan la importancia de las estructuras proposicionales, el texto y la interacción comunicativa desde una semántica en contexto. En algunos casos, como en el de Pinto (1996, 2001), se llega a proponer una compleja metodología de elaboración del resumen segmentando el texto en proposiciones y aplicando una serie de macrorreglas recursivas u operaciones de reducción de información semántica (supresión, generalización, construcción o integración, cero). 


\subsection{Enfoque centrado en el la figura del resumidor}

El enfoque procesual constituye una de las perspectivas básicas desde las que se puede orientar la investigación en el campo del resumen documental. Destaca la figura del resumidor y analiza cómo se enfrenta éste a la actividad resumidora desde la consideración de las distintas fases o estadios del proceso resumidor global y parcial. En un primer momento el proceso específico del resumen apenas es contemplado entre los tratadistas del resumen si bien se alude a un procedimiento general como un complejo modelo abstracto de análisis-síntesis del contenido de un documento original. Se pone el acento en la fase de lectura (comprensión) y en la de elaboración, mediante las operaciones de análisis-síntesis. Asimismo se destaca que entre el original y el resumen debe existir una relación de equivalencia semántica. Posteriormente encontramos modelos más elaborados de resúmenes en los trabajos de Lancaster (1996), Pinto (1995, 2001, 2004, 2006), Moreiro (2004) centrados más en lo que hace el resumidor y en los procesos cognitivos de "transferencia" o representación documental. Desde esta perspectiva el resumidor va asumiendo un papel activo y protagonista en el proceso global, se completan y profundizan las etapas del proceso del input textual, representación documental y evaluación. Asimismo, se defiende la recursividad de la operación de resumir y se incluye el papel del contexto como condicionante del procedimiento general. Por otra parte, esta aproximación cubrirá también los conceptos de relevancia, adecuación y representatividad como fenómenos cognitivos característicos de la recuperación de información. No obstante, el gran problema que cuestiona los estudios cognitivos del procesamiento de la información estriba en que una parte esencial del proceso tiene lugar en la mente del resumidor y por ello resulta difícil su medición objetiva. Asimismo, este enfoque únicamente cubre una parte del complejo fenómeno del resumen y no integra de un modo sistemático la interacción entre elementos cognitivos y los socioculturales. Sin embargo, y como fortalezas del método destacamos el concepto cognitivo de estrategia resumidora como factor fundamental para la práctica del resumen, así como el análisis pormenorizado de las distintas fases y competencias para lograr su desempeño eficaz.

El estudio del proceso resumidor puede acometerse bien analizando el proceso, de un modo directo o indirecto a través de herramientas procedentes de la psicología cognitiva, o con un enfoque alternativo a partir del análisis del producto.

a)El método más habitual para el estudio empírico del proceso resumidor es el análisis del protocolos verbales, un sistema de introspección a través de la verbalización en voz alta del pensamiento (Nardi y Fagundes 2007). Este sistema se complementa con otros como la observación directa de la conducta del resumidor o la retrospección ${ }^{2}$.

\footnotetext{
${ }^{2}$ Como ejemplo de esta aproximación metodológica centrada en el proceso Fujita $(2005,2007)$ enfoca los estudios de indización desde metodologías cognitivas de observación directa a través de análisis de protocolos verbales, tanto en indizadores expertos como noveles, para recabar información sobre procesos de metacognición que puedan aplicarse a la mejora de destrezas de lectura documental en textos científicos, como una de las fases de los procesos globales de indización. Desde una posición más pragmática el modelo de enseńanza propuesto por Fourie (2002) considera la perspectiva socio-cognitiva como idónea para la enseñanza de la indización y elaboración de resúmenes porque concibe a los usuarios como parte de
} 
Estos métodos son cuestionados debido a la influencia posible que puede ejercer el desarrollo del experimento sobre los sujetos sometidos a estudio. Creemos, además, que el análisis de protocolos verbales, si sólo atiende a cuestiones del texto y cotexto, podría conducir a estrategias restrictivas de resumen de carácter analítico que conllevarían a un alejamiento del concepto global de resumen así como del contexto de actividad en el que se inserta el proceso. Por otra parte, estos métodos cualitativos de aproximación cognitiva y metacognitiva a las tareas de resumen e indización han de cubrir todas las fases de las distintas operaciones del tratamiento documental de contenido, ya que estás se encuentran interconectadas para poder obtener una visión global y completa de los procesos de planificación estratégica, autorregulación de actividades y demás procesos metacognitivos implicados bien en el resumen, bien en la indización.

b) Complementariamente, podemos aproximarnos al estudio de la actividad resumidora desde el mismo texto, el resumen, ya que éste registra y plasma el resultado de las acciones y decisiones adoptadas por el resumidor para llegar, mediante su análisis, a conclusiones sobre el propio proceso.

\subsection{Enfoque socioconstructivista}

Desde finales de los años 80 , y sobre todo entrados los 90, comienza a cuestionarse el dominio del paradigma cognitivo en los procesos de organización del conocimiento y recuperación de información. La influencia de la teoría del dominio (Hjørland \&Albrechtsen,1995; Hjørland, 2002) y los postulados de Frohmann (1990, 1992) y Thellefsen $(2004,2006)$, configuran una dimensión sociocognitiva que se incorpora al tratamiento documental de contenido para lograr una teoría del resumen mas exhaustiva, concibiendo éste como un fenómeno socialmente situado.

El enfoque sociocultural incide sobre todos aquellos aspectos que rodean la actividad resumidora y resalta especialmente las circunstancias sociales y discursivo-culturales en las que se produce ésta, la figura del usuario como perteneciente a una comunidad discursiva y, finalmente, la importancia de los procesos de recepción del resumen documental desde el principio de aceptabilidad y adecuación a las convenciones socioretóricas de las distintas comunidades. A partir de esta hipótesis como complemento de las anteriores se construye un modelo resumidor de equivalencia semántica, estructural y pragmática, y se arbitra una red de competencias resumidoras locales y globales. Es pues una formulación que conjuga los aspectos formales, funcionales y comunicativos. En esta línea argumentativa se encuentran los estudios de Izquierdo Alonso $(2000,2003,2004$,$) quien considera la aproximación socio-cognitiva { }^{3}$ como

grupos de discurso o de dominio. Para eso sugiere que se planifiquen las actividades no sólo centradas en el contenido (enfoque orientado hacia el contenido) sino también en el contexto (concepción orientada hacia la demanda).

${ }^{3}$ La perspectiva cognitiva encuentra posiciones abiertamente críticas en y Hjorland (1995) y Frohmann (1999), entre otros. Estos declaran la necesidad de incorporar en la investigación psicológica una perspectiva social, cultural e histórica más amplia. Estas discrepancias y otras similares originaron en los últimos años del siglo XX nuevas corrientes teóricas en la ciencia de la información, cuya característica fundamental es la importancia que se concede a lo social, lo cultural y lo contextual. 
un marco propicio para la enseñanza de la indización y elaboración de resúmenes. Desde sus propuestas, incorpora una dimensión pragmático-comunicativa al resumen documental y se aproxima al concepto de resumen y de la actividad resumidora desde una integración del contexto (situaciones comunicativas), los procesos cognitivo-sociales y el objeto/texto en su dimensión social de género discursivo.

\section{4.- Enfoque comunicativo y sociocultural como puente integrador}

Para el estudio del resumen y de su actividad consideraremos todas las variables semiótico-textuales, la teoría cognitiva y funcional para el estudio de los procesos, y la configuración del contexto como manifestación del acto comunicativo-documental. Atenderemos, pues, tanto al entorno o condiciones en las que se crea el texto objeto de resumen como al contexto documental para la representación del mismo. Valoraremos la situacionalidad como uno de los factores clave que hacen que un texto/resumen sea relevante y aceptable en una situación de ocurrencia real o potencial determinada (configuración contextual específica). Así, dentro del proceso general resumidor, consideraremos los distintos tipos específicos de texto, analizaremos la función y objetivos de la demanda resumidora acorde con las exigencias generales de eficacia comunicativo-documental y los parámetros socioculturales del contexto. Pero los textos o cualquier otro inputs a resumir, no han de verse como una variable aislada del proceso resumidor sino como un elemento más a tener en cuenta dentro de un conjunto de variables significativas en el contexto de producción y difusión de los resúmenes documentales. En este sentido, integramos el texto dentro de su proceso, examinando las distintas actividades secuenciadas e interdependientes que responden a objetivos y funciones específicas de realización del resumen así como las estrategias para llevarlo a desde una dimensión social y situada. Por último consideramos todo el proceso, y el mismo resumen como producto final convencionalizado, desde el complejo sistema interactivo de las comunidades discursivas y sus prácticas sociales.

\section{Valoraciones finales}

Para concluir este trabajo realizaremos una serie de consideraciones globales que agrupan las afirmaciones más importantes del estudio y subrayan el gran potencial de investigación que encierra el fenómeno del resumen documental en toda su complejidad.

- Los estudios del resumen documental se caracterizan por perspectivas alternativas que afectan al texto como materia prima del resumen y al contexto de la actividad resumidora y que tienen sus orígenes en intereses diversos y en la adopción de enfoques metodológicos alternativos o complementarios, con diferenciación de ejes y prioridades entre ellos.

- Parte de la complejidad del resumen documental reside en su carácter multidimensional. Desde este amplio abanico podemos hacer referencia a una teoría global de los estudios sobre resumen documental con varias facetas de análisis desde las que poder abordar sus aspectos generales y parciales. El enfoque integrador del resumen, 
incluirá tanto la dimensión producto-proceso (caracterizándolo como símbolo semiótico, proceso situado social y pragmáticamente, y acto de comunicación documental) como la conjunción complementaria de enfoques para construir una teoría general del resumen y del proceso resumidor. En este sentido podemos construir una teoría comprehensiva del resumen documental, fundamentada en tres dimensiones interconectadas: una dimensión gnoseológica, otra procedimental y estratégica, referida al saber técnico, y una última creativa, validada por la experiencia personal y profesional de resumidor, desde su intuición y habilidades creativas conjugadas con las normas de referencia profesional e institucional de cada uno de los servicios de resumen.

- Dentro de las perspectivas de análisis del resumen documental y su actividad resumidora hemos de tener en cuenta que éste es algo más que un proceso de transcodificación documental o meta-representación. Constituye una operación semiótica-textual, un acto comunicativo con una función eminentemente documental (lo que le dota de especificidad frente a otros tipos de resúmenes), una actividad en la que intervienen sujetos y que se desarrolla en un contexto social determinado o dominio de especialización. Esto conlleva una complejidad en este tipo de estudios y determina la inclusión de distintos elementos en la teoría resumidora: las variables documentales, lingüísticas, sociocomunicativas, cognitivas y la instrumentalidad tecnológica. Asimismo ha de contemplarse el estudio del resumen documental como producto y como proceso (en sus relaciones internas y externas). Las relaciones internas o endofóricas son intertextuales entre el texto origen y el resumen resultante. Las relaciones exofóricas o externas vienen determinadas por la importancia del usuario de información destinatario del resumen, los condicionantes del proyecto resumidor por parte del sistema de información, los procesos mentales implicados en la representación documental, los procesos socioculturales en la recepción del resumen documental, la adquisición de competencia resumidora, etc. Construiríamos con esta confluencia de niveles de análisis una teoría global del resumen documental que atendiera al proceso, al producto y a la adecuación funcional del resumen documental en la cultura receptora.

- Las teorías sobre el resumen documental son bastante recientes. Es a partir de fines de los 80 cuando surge un interés por analizar de un modo sistemático las diferentes vertientes del resumen documental, desde distintos ángulos de aproximación, reafirmándose este tipo de estudios, con carácter propio, dentro del tratamiento documental de contenido. Podemos categorizar los enfoques en cuatro frentes: epistémicos, cognitivos, sociodiscursivos y lingüísticos. Se caracterizan por perspectivas alternativas sobre el propio concepto de texto y el contexto de la actividad resumidora, ya que tienen sus orígenes en intereses diversos con diferenciación de ejes metodológicos. El reto al que se enfrentan este tipo de estudios es encontrar un modo para integrar las teorías existentes, sin renunciar a toda la riqueza de perspectivas y visiones que ofrecen cada una de ellas, configurando un mapa consolidado y plural de modelos y estrategias resumidoras, de modo que podamos abordar eficazmente los procesos de representación documental en distintos ámbitos de especialidad.

- Los distintos modelos ayudarán a describir y explicar las reglas que ayuden a comprender y hacer eficaz el proceso de resumen documental, formulando estrategias para enfrentarnos a los diferentes problemas de esta operación de análisis de contenido y a coordinar todos los aspectos y variables que intervienen en ella. Nos marcamos, pues, 
como meta: a) construir de un modo integrado y desde un marco interdisciplinario de análisis el aparato conceptual adecuado que defina y explique todos los fenómenos relacionados con el resumen documental en contextos de especialización más allá del tradicional resumen científico; b) describir y explicar la actividad resumidora, clarificando y clasificando los diversos fenómenos, buscando probabilidades, regularidades, estrategias y principios; c) desarrollar una didáctica resumidora que garantice una buena praxis y consolide una competencia adecuada para el profesional.

Hemos intentado sistematizar en modelos de investigación las distintas líneas posibles de investigación que cubren los estudios actuales de esta operación de representación documental. Son muchos los logros pero aún quedan muchos problemas por resolver y nuevos retos a conquistar relacionados con los resúmenes en contextos especializados, las diferentes manifestaciones semióticas objeto de representación documental, los procesos de automatización o la mejora en la formación y el aprendizaje del resumen documental.

\section{Referencias Bibliográficas}

BORKO H\& BERNIER CL (1975): Abstracting concepts and methods. New York: Academic Press.

CLEVELAND, D. \& A. CLEVELAND (1983). Introduction to Indexing and Abstracting. Edition. Englewood, CO: Libraries Unlimited

CREMMINS, ET. (1982): The art of abstrating. Philadelphia: ISI

FOURIE, I. (2003): How can we take a socio-cognitive approach in teaching indexing and abstracting? The Indexer, vol. 23, no 2, p. 83-85.

FROHMANN, B. (1990). Rules of Indexing: A Critique of Mentalism in Information Retrieval Theory. Journal of Documentation, 46(2), 81-101.

FROHMANN, B. (1992). The Power of Images: A Discourse Analysis of the Cognitive Viewpoint. Journal of Documentation, 48(4), 365-386.

FUJITA, M. S. L (2007): La enseñanza de la lectura documentaria en el abordaje cognitivo y socio-cognitivo: orientaciones a la formación del indizador. Anales de Documentación, 2007 (10), pp. 397-412

FUJITA, M. S. L.; CERVANTES, B. M. N (2005): Abordagem cognitiva do protocolo verbal na confirmação de termos para a construção de linguagem documentária em inteligência competitiva. In: VALENTIM, Marta Lígia Pomim (coord): Métodos qualitativos de pesquisa em ciência da informação. São Paulo.

HJØRLAND, B (1997). Information seeking and subject representation: an activity-theoretical approach to information science. Westport: Greenwood Press, 213 p.

HJØRLAND, B (2002a): Epistemology and the socio-cognitive perspective in information science. Journal of the American Society for Information Science and Technology, 53 (4), pp. 257-70.

HJØRLAND, B (2002b). Domain analysis in information science: Eleven approaches - traditional as well as innovative. Journal of Documentation, 58, p. 422-462. 
HJORLAND, Birger \& ALBRECHTSEN, Hanne (1995): Toward a new horizon in information science: Domain analysis. Journal of the American Society for Information Science, 46(6), 400-425.

IZQUIERDO ALONSO (2004). M: Nuevos retos en el análisis documental de contenido. SCIRE: representación y organización del conocimiento, 10 (1), 31-50.

IZQUIERDO ALONSO, M (2000): Nuevos enfoques en el estudio del tratamiento documental de contenido desde los presupuestos de las ciencias del lenguaje. En: Revista SCIRE, vol 6 (1), pp.143-163

IZQUIERDO ALONSO, M (2003): Procesamiento pragmático para el tratamiento documental de contenido. Documentación de las Ciencias de la Información, no 26, pp. 181-198

IZQUIERDO ALONSO, M; MORENO FERNÁNDEZ, LM (2009): El resumen documental: un reto didáctico. Madrid, ANABAD.

IZQUIERDO ARROYO, J M (1990): Teoría del resumen. En: Esquemas de Lingüistica documental. Barcelona, PPU, pp. 465-482.

IZQUIERDO ARROYO, J.M. (1993): De la Semiótica del discurso a la Semiótica Documental. En J. A. Moreiro: Aplicación de las Ciencias del Texto al Resumen Documental. Madrid, Universidad Carlos III / BOE, 199-216

LANCASTER, F. W (1996). Indización y resúmenes: teoría y práctica. Buenos Aires: EB Publicaciones

LÓPEZ YEPES, J.; SAGREDO FEPNÉNDEZ, (.et al) (1981): Estudios de Documentación general e informativa, Madrid, Seminario Millares Carlo.

MONTESI, M (2006): Métodos de evaluación y calidad de resúmenes documentales. Gijón, TREA

MOREIRO GONZÁLEZ, JA (1993): Aplicación de las Ciencias del Texto al Resumen Documental. Madrid, Universidad Carlos III / BOE, 1993.

MOREIRO GONZÁLEZ, JA (2004): El contenido de los documentos textuales: su análisis y representación mediante el lenguaje natural. Gijón, TREA

NARDI, M.I.A. y FAGUNDES, S.A.F.(2003): Observing documentary reading by verbal protocol. Information Research, v. 8, no 4, paper no. 155 Disponible en: <http://informationr. net/ir/8-4/paper155.html>. [Consultado: 27/04/2009].

PINTO M; GALVEZ, C, (1996): Análisis documental de contenido: procesamiento de la información. Madrid, Síntesis

PINTO, M (1991): Análisis documental: fundamentos y procedimientos. Madrid, EUDEMA

PINTO, M. (1995): Documentation abstracting: toward a methodological model. Journal of the American society for information science, 46(3), pp 225-234

PINTO, M. (2003): Abstracting/Abstract Adaptation to Digital Environments: Research Trends. Journal of Documentation, vol. 59(5), pp, 581-608.

PINTO, M. (2006): A grounded theory on abstracts quality: weighting variables and attributes. Scientometrics, vol. 69(2), pp. 213-226

ROWLEY, J. (1982). Abstracting and Indexing. London: Clive Bingley.

THELLEFSEN, T.B \& THELLEFSEN, M. (2003). Problems concerning the process of subject analysis and the practice of indexing : a semiotic and semantic approach towards user oriented needs in document representation and information searching. Semiotica, 144(1), $177-218$

VAN DIJK, T.A (1978): La ciencia del texto: un enfoque interdisciplinario. Barcelona, Paidos, 1983 
VAN DIJK, T.A. (1980). Macroestructures. An interdisciplinary study of global structures in discourse, interaction and cognition. Hillsdale, NJ: Erlbaum.

VAN DIJK, T.A., KINTSCH, W. (1983). Strategies of discourse comprehension. New York: Academic Press 